\title{
Bias, precision and accuracy in the estimation of cuticular and respiratory water loss: A case study from a highly variable cockroach, Perisphaeria sp.
}

\author{
Emilie M. Gray ${ }^{1}$, Steven L. Chown* \\ Department of Botany and Zoology, Centre for Invasion Biology, Stellenbosch University, Private Bag X1, Matieland 7602, South Africa
}

Received 21 June 2007; received in revised form 27 August 2007; accepted 28 August 2007

\begin{abstract}
We compared the precision, bias and accuracy of two techniques that were recently proposed to estimate the contributions of cuticular and respiratory water loss to total water loss in insects. We performed measurements of $\dot{V} \mathrm{CO}_{2}$ and $\dot{V} \mathrm{H}_{2} \mathrm{O}$ in normoxia, hyperoxia and anoxia using flow through respirometry on single individuals of the highly variable cockroach Perisphaeria sp. to compare estimates of cuticular and respiratory water loss (CWL and RWL) obtained by the $\dot{V} \mathrm{H}_{2} \mathrm{O}-\dot{V} \mathrm{CO}_{2} y$-intercept method with those obtained by the hyperoxic switch method. Precision was determined by assessing the repeatability of values obtained whereas bias was assessed by comparing the methods' results to each other and to values for other species found in the literature. We found that CWL was highly repeatable by both methods $(R \geqslant 0.88)$ and resulted in similar values to measures of CWL determined during the closed-phase of discontinuous gas exchange (DGE). Repeatability of RWL was much lower $(R=0.40)$ and significant only in the case of the hyperoxic method. RWL derived from the hyperoxic method is higher (by $0.044 \mu \mathrm{mol} \mathrm{min}{ }^{-1}$ ) than that obtained from the method traditionally used for measuring water loss during the closed-phase of DGE, suggesting that in the past RWL may have been underestimated. The very low cuticular permeability of this species $\left(3.88 \mu \mathrm{g} \mathrm{cm}^{-2} \mathrm{~h}^{-1} \mathrm{Torr}^{-1}\right)$ is reasonable given the seasonally hot and dry habitat where it lives. We also tested the hygric hypothesis proposed to account for the evolution of discontinuous gas exchange cycles and found no effect of respiratory pattern on RWL, although the ratio of mean $\dot{V} \mathrm{H}_{2} \mathrm{O}$ to $\dot{V} \mathrm{CO}_{2}$ was higher for continuous patterns compared with discontinuous ones.
\end{abstract}

(C) 2007 Elsevier Ltd. All rights reserved.

Keywords: Discontinuous gas exchange; Metabolic rate; Repeatability; Water balance

\section{Introduction}

Dehydration is a major challenge facing all terrestrial organisms and it is especially pronounced for small animals such as arthropods. Insects respond to desiccation in a variety of ways (Hadley, 1994a; Chown and Nicolson, 2004; Alpert, 2006), and over longer time periods, differences among populations and species in tolerance of partial desiccation, resistance to desiccation, and initial body water contents may all evolve differentially to

\footnotetext{
*Corresponding author. Tel.: + 27218082385 ; fax: + 27218082995.

E-mail address: slchown@sun.ac.za (S.L. Chown).

${ }^{1}$ Current address: Department of Biological Sciences, University of Notre Dame, Notre Dame, IN 46556-0369, USA.
}

prolong survival time under dry conditions. Indeed, such differences have been documented in comparisons among species and populations at several spatial scales (Chown, 2002; Hoffmann et al., 2003), and have also been shown to evolve rapidly in the laboratory (Gibbs et al., 1997; Telonis-Scott et al., 2006).

Despite widespread documentation of apparently adaptive patterns in insect desiccation resistance, and considerable understanding of the mechanisms underlying insect water balance (reviewed in Hadley, 1994a; Chown and Nicolson, 2004), the contributions of cuticular and respiratory water loss to dehydration, and the significance of the mechanisms by which each may be modulated, remain highly contentious. The relative contributions of these major avenues of water loss are known for less than 
30 insect species (Chown, 2002; Schilman et al., 2005), representing only four orders. This paucity of estimates of cuticular water loss rate (CWL) and respiratory water loss rate (RWL) mean that even fundamentally significant features of water balance, such as the relative contributions of CWL and RWL to total water loss and the ways in which RWL is modulated remain poorly understood (Kestler, 1985; Hadley, 1994b; Lighton, 1998; Chown, 2002). Some studies have concluded that RWL is indeed a significant component of the evolved response to dry conditions (e.g., Gibbs et al., 2003; Duncan, 2003; Benoit and Denlinger, 2007), and that modulation of metabolic rate and gas exchange pattern are important ways in which RWL might be altered (e.g., Zachariassen et al., 1987; Duncan et al., 2002; Chown and Davis, 2003; Marais et al., 2005; White et al., 2007). By contrast, other studies have suggested that RWL is so small that it is of minor evolutionary significance (e.g., Lighton et al., 1993; Quinlan and Hadley, 1993; Hadley 1994b). Moreover, the significance of modulation of metabolic rate or gas exchange pattern as a means to effect changes in RWL has also been questioned (Djawdan et al., 1998; Bradley et al., 1999; Bosch et al., 2000; Rourke, 2000; Shelton and Appel, 2001). Quinlan and Lighton (1999) concluded that ‘... interrelationships between gas exchange and water balance are still largely a matter of conjecture'.

Perhaps the major reason for the present state of the field is that the measurement of CWL and RWL or their distinction in estimates of total water loss are not simple matters. Traditionally, CWL has been measured using gravimetric, isotopic or electronic techniques, applied to whole insects, or portions of their cuticle (in vivo or in vitro), all of which have their problems (reviewed in Hadley, 1994a; Chown and Nicolson, 2004). Estimates of RWL have been even more problematic. Typically, they have been restricted to insects in which discontinuous gas exchange (DGE; see Chown et al., 2006a) means that for some proportion of the time the spiracles are closed, allowing cuticular and respiratory water loss to be distinguished either by gravimetric means or by electronic techniques (Machin et al., 1991; Lighton, 1992; Lighton et al., 1993; Quinlan and Hadley, 1993).

Recently, two techniques have been developed which seem set to change the ease with which estimates of RWL and CWL can be obtained. In the first approach, a regression of $\mathrm{H}_{2} \mathrm{O}$ against $\dot{V} \mathrm{CO}_{2}$ and extrapolation to the $y$-intercept allows estimation of CWL by assuming that in the absence of gas exchange (and easily identifiable excretion events) water loss must be entirely, or predominantly, cuticular (Gibbs and Johnson, 2004). The second approach (Lighton et al., 2004) relies on spiracular closure, or considerable reduction in spiracle cross-sectional area, following exposure of insects to extreme hyperoxia. Using $\mathrm{CO}_{2}$ as a trace gas and by directly measuring $\mathrm{H}_{2} \mathrm{O}$, an estimate of CWL can be obtained when the spiracles are either closed or nearly so. Both sets of proponents claim that their techniques provide a useful means of distinguish- ing CWL from RWL and estimating their values. In the former case, these claims were based on a comparison of respiratory exchange ratio of $\mathrm{CO}_{2}: \mathrm{H}_{2} \mathrm{O}$ with a single other study (Gibbs and Johnson, 2004), though with no explicit mention of absolute rates of CWL and RWL. In the latter, a somewhat more extensive comparison was made between the focal species and published values either of cuticular permeabilities (CP) or proportional water loss (Lighton et al., 2004, p. 4469).

However, these assessments of the validity of the techniques only considered bias (and then not especially carefully in one case): the likelihood that the estimated values were close to the true values of these parameters. Neither study made any attempt to assess precision, or the variance of the estimation procedure (see Walther and Moore, 2005). A later study, by Schilman et al. (2005), did compare the methods by inspection of the coefficient of variation of $\mathrm{CP}$ for each method. However, because only single estimates were made per individual for each measurement technique, these coefficients of variation represent an estimate of population variation rather than an estimate of precision, with the additional complication that groups of ants, rather than individuals were used. Nonetheless, Schilman et al. (2005) pointed out that the regression method results in substantially more variability than the hyperoxic switch method, and that the former occasionally produces uninterpretable results. How general these findings are, especially for individual insects, is not known.

In consequence, the present situation is that two techniques exist which have both been hailed as potentially useful for routinely estimating CWL and RWL in resting or active insects. However, the precision of these techniques is unknown and their bias has been inadequately assessed. In consequence, the accuracy of the techniques, or their performance in repeatably estimating the true value of the parameters of interest (CWL, RWL, and their derivatives such as CP), remains poorly established. Here, we address this problem in several ways. First, we investigate the precision of both methods by performing repeated measures of the same individuals on different days. We do so using a cockroach species (Perisphaeria sp.) with highly variable gas exchange patterns (Marais and Chown, 2003) because this enables DGE-based estimates to be compared with estimates derived from other gas exchange patterns using other techniques, and because a finding of high precision in such a variable species would suggest that in species with less variation (likely many species, see Chown, 2001; Marais et al., 2005) the techniques will also be precise. Second, to assess the bias of the methods, we compare the estimates of CWL and RWL obtained by these two methods to each other, and to estimates based on the more traditional method of using the closed-phase of DGE to estimate CWL (see e.g. Quinlan and Hadley, 1993). We also compare the different estimates of the contributions of CWL and RWL to total water loss and estimates of CP of this Perisphaeria species 
to the data available in the literature. Finally, owing to the variability of gas exchange patterns in our study species, we determine the extent to which DGE results in reduced water loss by comparison with continuous ventilation-i.e., we provide a test of the hygric hypothesis proposed to account for the maintenance of DGE (Chown et al., 2006a).

\section{Materials and methods}

\subsection{Experimental animals and design}

Individuals of the Perisphaeria sp. (Blattodea, Blaberidae) were collected between flat rocks at the top of Stellenbosch Mountain (altitude $>950 \mathrm{~m}, 33^{\circ} 57^{\prime} \mathrm{S}$, $18^{\circ} 53^{\prime} \mathrm{E}$ ). In the laboratory, cockroaches were kept in 1.81 plastic containers at a maximum density of six individuals per container, with pieces of egg carton to create hiding spaces. Experience has shown that individuals of this species are most comfortable in the company of conspecifics, but it is also known that high densities in Periplaneta americana result in elevated $\mathrm{CP}$ as a consequence of cuticular damage (Machin and Lampert, 1987). The cockroaches had access to apple slices and dry cat food. Wet cotton wool maintained humidity in each container and the containers were held in a temperaturecontrolled chamber (Labcon, CA, USA) at $21 \pm 1^{\circ} \mathrm{C}$ and with a $12 \mathrm{~h}: 12 \mathrm{~h}$ L:D cycle. Individual cockroaches were identified by small markings made with non-toxic paint. They were held under these conditions for at least 6 weeks before experiments commenced to exclude possible variation due to habitat differences (Marais and Chown, 2003) and to identify individuals parasitized by rhipiphorid beetles, which were excluded.

Each cockroach was isolated in a Petri dish with wet filter paper for $24 \mathrm{~h}$ prior to measurements, then weighed immediately before and after each trial on a Mettler Toledo AX504 analytical balance (accuracy $0.1 \mathrm{mg}$, Mettler, Columbus, OH, USA). An experimental trial consisted of measuring the second-to-second $\dot{V} \mathrm{CO}_{2}$ and $\dot{V} \mathrm{H}_{2} \mathrm{O}$ of an individual cockroach for $3.5 \mathrm{~h}$ in air (normoxia) (see Chown, 2001 for rationale), then for $20 \mathrm{~min}$ in pure oxygen (hyperoxic switch) and finally for 5-15 min in pure nitrogen (anoxic switch). The latter was done because Lighton et al. (2004) suggested that this technique might yield information on maximal degree of spiracular opening and properties of the tracheal system. Thus, our approach enabled us to identify the precision of this method too. This amount of time in nitrogen was sufficient to knock down the cockroaches but permitted their full recovery within a few minutes after being returned to normoxia.

For each trial, the air $\left(21 \% \mathrm{O}_{2}\right.$, balance $\left.\mathrm{N}_{2}\right)$ obtained from a tank was first stripped of residual water and $\mathrm{CO}_{2}$ by passage through columns containing soda lime, silica gel and Drierite ${ }^{\circledR}$ (Krugersdorp, South Africa). Its flow rate was then precisely controlled to $200 \mathrm{ml} \mathrm{min}^{-1}$ by a mass flow controller (Sidetrack, Monterey, CA, USA). Follow- ing this, the gas was pushed through a $5 \mathrm{ml}$ chamber containing the animal and on to a Li-Cor Li-7000 infrared gas analyzer (Lincoln, NE, USA), which was used to measure the $\mathrm{CO}_{2}$ and $\mathrm{H}_{2} \mathrm{O}$ content of the air, and values were recorded each second using the associated software. Bev-A-Line tubing was used throughout the system to minimize errors linked to water absorption. The animal chamber, made of glass and stainless steel mesh filters, was cleaned between each trial using ethanol. The chamber was placed inside a Sable Systems International (Las Vegas, NV, USA) AD-1 activity detector to detect movements of the cockroach during the experiment, and the data were simultaneously recorded via an auxiliary input of the Li-7000. The entire setup was housed in a temperaturecontrolled chamber at $21 \pm 1{ }^{\circ} \mathrm{C}$ with lights on. During the experiment, the cockroach was in dim light due to its placement in the activity detector. All experiments were performed during light hours, between 09:00 and 18:00 h, although time of day was not entered into the models because it is typically not an important component of variation in gas exchange parameters in this species (Marais and Chown, 2003).

Fifteen cockroaches, named ' $a$ ' thru ' $o$ ' were used in this study. Three individuals $(k, l$ and $m$ ) were identified as significant outliers in the data (i.e., lying more than two standard deviations beyond the other data). Therefore, we only included them in those analyses where precision across the entire sample was estimated. Data from the remaining 12 cockroaches were used for the more extensive analyses, thus avoiding the potentially confounding effects of the outlier data. Five experimental trials, each separated by a period of 6-7 days, were performed on each cockroach. The interval between repeated measures is important because the shorter it is the greater the likelihood that high precision (or repeatability) will be found (Chappell et al., 1996; Bech et al., 1999). In using the 6-7 days interval, we purposefully weighted the outcome against high precision to effect as conservative an estimate of precision as possible without including too large an effect of ageing. Age is known to alter many physiological characteristics in insects (e.g. Terblanche et al., 2004), but we considered a month in the life of these typically longlived cockroaches a reasonably short period (see additional discussion in Marais and Chown, 2003).

\subsection{Data extraction and initial analysis}

Data were extracted using Expedata version 1.0.16 (Sable Systems International, Las Vegas, NV, USA) and were analyzed with R version 2.3.1 (http://www.r-project. org) or SAS version 9.1 (SAS Institute Inc., Cary, NC, USA). Previously, Marais and Chown (2003) found that individuals of this species exhibit a variety of gas exchange patterns while at rest: discontinuous gas exchange cycles (DGC), burst-interburst (BI; similar to DGC but lacking a flutter or F-phase), pulsation, and continuous (C). In a few trials, episodes of the pulsation pattern described in 
Marais and Chown (2003) were found, but this pattern was never dominant. Thus, for each trial we assessed whether the pattern of gas exchange was continuous $(\mathrm{C})$, burst-interburst (BI) or discontinuous (DGC) following the convention developed in Marais et al. (2005). The assessment was performed over the last $90 \mathrm{~min}$ of normoxia (i.e., after the animal had been in the cuvette for $2 \mathrm{~h}$ ). Briefly, a trace was considered continuous if greater than $30 \%$ of the $\mathrm{VCO}_{2}$ values were above the midline of the $\dot{V} \mathrm{CO}_{2}$ trace. DGC and $\mathrm{BI}$ were distinguished based on the presence or absence of an F-phase, respectively. Although several patterns sometimes occurred in one trial, we qualified a trial by the pattern that occurred for the longest duration within the 90-min interval observed. For all types of gas exchange, we recorded mean $\dot{V} \mathrm{CO}_{2}$ and $\mathrm{VH}_{2} \mathrm{O}$ typically over a period of at least $1 \mathrm{~h}$ of inactivity. In the case of BI and DGC, we measured the duration and emission rate of each cycle phase and obtained a mean over five cycles. We consider a single cycle insufficient to represent the likely variation found in a gas exchange trace, hence our focus on the $90 \mathrm{~min}$ period. Measurement of a single cycle would mean that cycle variation and individual variation are identical, so confounding estimates of repeatability.

In the case of DGC and BI patterns, RWL and CWL were calculated as suggested by Quinlan and Hadley (1993). Water loss rate during the C-phase was taken to be equivalent to $\mathrm{CWL}$, whereas mean water loss over the entire gas exchange cycle (for several full cycles) provided an estimate of mean $\dot{V}_{2} \mathrm{O}$. The difference between the two values provided an estimate of mean RWL.

Following Gibbs and Johnson (2004), we calculated the $y$-intercept and slope of the regression of $\dot{V} \mathrm{H}_{2} \mathrm{O}$ against $\dot{V} \mathrm{CO}_{2}$. The slope was analyzed because Gibbs and Johnson (2004) claim that the slope of the regression represents the hygric cost of gas exchange. Although significance and other parameters of these regressions are likely to be in error owing to temporal autocorrelation of the data and extrapolation beyond the scope thereof in cases where the spiracles do not close (see Quinn and Keough, 2002), we ignored these problems for the purposes of the test. We return to them in Section 4. The slope and intercept values were calculated over time periods of 90,60 and $30 \mathrm{~min}$ (full period, $2 / 3$ and $1 / 3$ thereof) to determine whether there is an effect of duration on precision of the estimates of the slope and $y$-intercept. In addition, we compared the slopes and intercepts calculated for the first $30 \mathrm{~min}$ and the last $30 \mathrm{~min}$ of the $90 \mathrm{~min}$ interval to determine whether it is worth waiting for as long as possible to ascertain these values given the notorious lethargy of water in flow-through gas exchange systems. The slope and $y$-intercept estimates derived from the last $30 \mathrm{~min}$ interval were used for statistical assessments of the effect of gas exchange pattern and calculation method on various parameters (such as CWL).

After the hyperoxic switch, cockroaches sometimes carried on with somewhat continuous gas exchange. However, in most cases we found a C-phase (as continuous breathers stopped releasing $\mathrm{CO}_{2}$ ). We noted $\dot{V} \mathrm{CO}_{2}$ and $\dot{V} \mathrm{H}_{2} \mathrm{O}$ during the hyperoxia-induced C-phase (hereafter, hypC-phase). Lighton et al. (2004) calculated two estimates of RWL (one conservative and one upper limit) for the hypC-phase because of limited temporal resolution, error linked to group measurements and lack of complete spiracular closure. Since we were not faced with these possible sources of error, and always used a period of zero or near zero $\mathrm{VCO}_{2}$ for estimating RWL, we obtained RWL directly from the difference between mean $\dot{V}_{2} \mathrm{O}$ and hypC- $V \mathrm{H}_{2} \mathrm{O}$ (which is considered CWL). These estimates of RWL and CWL were then used in statistical comparisons. For CP, surface area was calculated according to Edney and McFarlane (1974) by Meeh's formula: SA $\left(\mathrm{cm}^{2}\right)=12 \times$ body mass $(\mathrm{g})^{0.63}$.

Following $20 \mathrm{~min}$ in hyperoxia insects were switched to $100 \% \mathrm{~N}_{2}$ and left in the presence of flowing nitrogen for up to $15 \mathrm{~min}$ or a shorter period if they showed a large $\mathrm{CO}_{2}$ and $\mathrm{H}_{2} \mathrm{O}$ burst accompanied by a short burst of activity (which corresponded to muscle spasms and loss of motor control, as visually assessed). The peak water loss rate value was recorded for each animal. As soon as the peak had occurred the recording was stopped and the animal was returned to a Petri dish with humid filter paper. Cockroaches always recovered within $5 \mathrm{~min}$. In cases where no peak was found within $15 \mathrm{~min}$ of anoxia, the animals were returned to normoxia to prevent permanent injury. Peak $\mathrm{VH}_{2} \mathrm{O}$ was used to assess the precision of this method for estimating spiracular opening.

\subsection{Statistical analyses}

To determine the precision of each method for CWL and RWL estimation, as well as the precision of mean $\dot{H} \mathrm{H}_{2} \mathrm{O}$ and anoxic $\mathrm{H}_{2} \mathrm{O}$ burst, repeatability was calculated as described in Lessells and Boag (1987). Because we were interested in precision, body mass of each individual was included as a covariate in the ANOVAs and removed if its effect was not significant. Confidence intervals of repeatability were calculated following the methods of Krebs (1999) using the appropriate degrees of freedom for each variable to obtain the $F$-statistics at $p=0.05$. Standard errors of repeatability were calculated following Becker (1984).

Following estimates of precision we compared the absolute values of CWL, RWL, CP and \% RWL among the three methods to ascertain their likely bias. This comparison was made using repeated measures ANOVA, implemented using the mixed procedure of SAS, because the same individuals were used in estimates of these parameters using each of the three methods. In doing so we assumed that the DGC-based method, accepted to date in the literature (see reviews in Hadley, 1994b; Chown, 2002), would serve as an acceptable benchmark. However, CP estimates from this study and those provided by Lighton et al. (2004) and Schilman et al. (2005) were also compared with data obtained from the literature (especially 
Hadley, 1994a). The slopes and $y$-intercepts obtained from all four-time intervals were also compared by repeated measures ANOVA.

Finally, the effect of gas exchange pattern on $\dot{V} \mathrm{CO}_{2}$, $\dot{V} \mathrm{H}_{2} \mathrm{O}$, hypC-phase $\dot{V} \mathrm{H}_{2} \mathrm{O}, \% \mathrm{RWL}$, and slope and intercept of the $\dot{V} \mathrm{CO}_{2}$ and $\dot{V} \mathrm{H}_{2} \mathrm{O}$ regressions was assessed by restricted maximum likelihood (REML) ANOVA. The linear mixed-effects models included body mass, pattern and their interaction as fixed effects and individual as a random effect. We fitted body mass before pattern to correct initially for any effect of body mass. Body mass was eliminated from the model if its effect was non-significant. All data were $\log _{10}$-transformed to achieve normality. The REML ANOVA performed in $R$ tests the significance of the fixed factors after accounting for the random effect of individual. We did not use repeated measures ANOVA because, although measurements were repeated each week on the same individuals, the pattern that was observed could not be predicted and that is the independent variable of interest in our analyses. Nonetheless, we included individual identity in the models to retain non-independence. To specifically test the hygric hypothesis, we extracted from the raw dataset individuals that had shown continuous patterns on some repeats and DGC on other repeats (eight individuals) and compared RWL and CWL (as absolute and relative values) between both patterns.

\section{Results}

Of the 15 individuals examined in this study, only two individuals showed the same gas exchange pattern on all five repeats (DGC in both cases); the other individuals showed either two (nine individuals) or three (four individuals) different patterns (Fig. 1). Of the patterns found, BI was encountered least often (Table 1), and the pulsation pattern recorded by Marais and Chown (2003) was never identified as dominant for an individual. The classification method we adopted necessarily meant that some traces, which might at first appear somewhat noncontinuous, are actually continuous (Fig. 1C). Obviously, setting the criteria less conservatively would provide a different assessment, but this did not affect the outcome of our assessments to any large degree. Following the hyperoxic switch, hypC-phases were encountered on most but not all repeats ( $88 \%$ of all repeats), at variable times following the switch to hyperoxia and for variable durations (data not shown). In some cases, individuals continued to release $\mathrm{CO}_{2}$ at variable rates throughout the hyperoxic episode and were excluded from further analyses. Anoxia resulted in a large burst of $\mathrm{CO}_{2}$ and $\mathrm{H}_{2} \mathrm{O}$ release in most trials (Table 1), and would probably have occurred in all cases had the anoxic period extended beyond $15 \mathrm{~min}$. However, we preferred to minimize stress and guarantee the survival of individuals by limiting anoxic exposure. When using the regression method, four trials out of 60 were unsuitable for inclusion because the $y$-intercepts resulted in negative values of RWL.
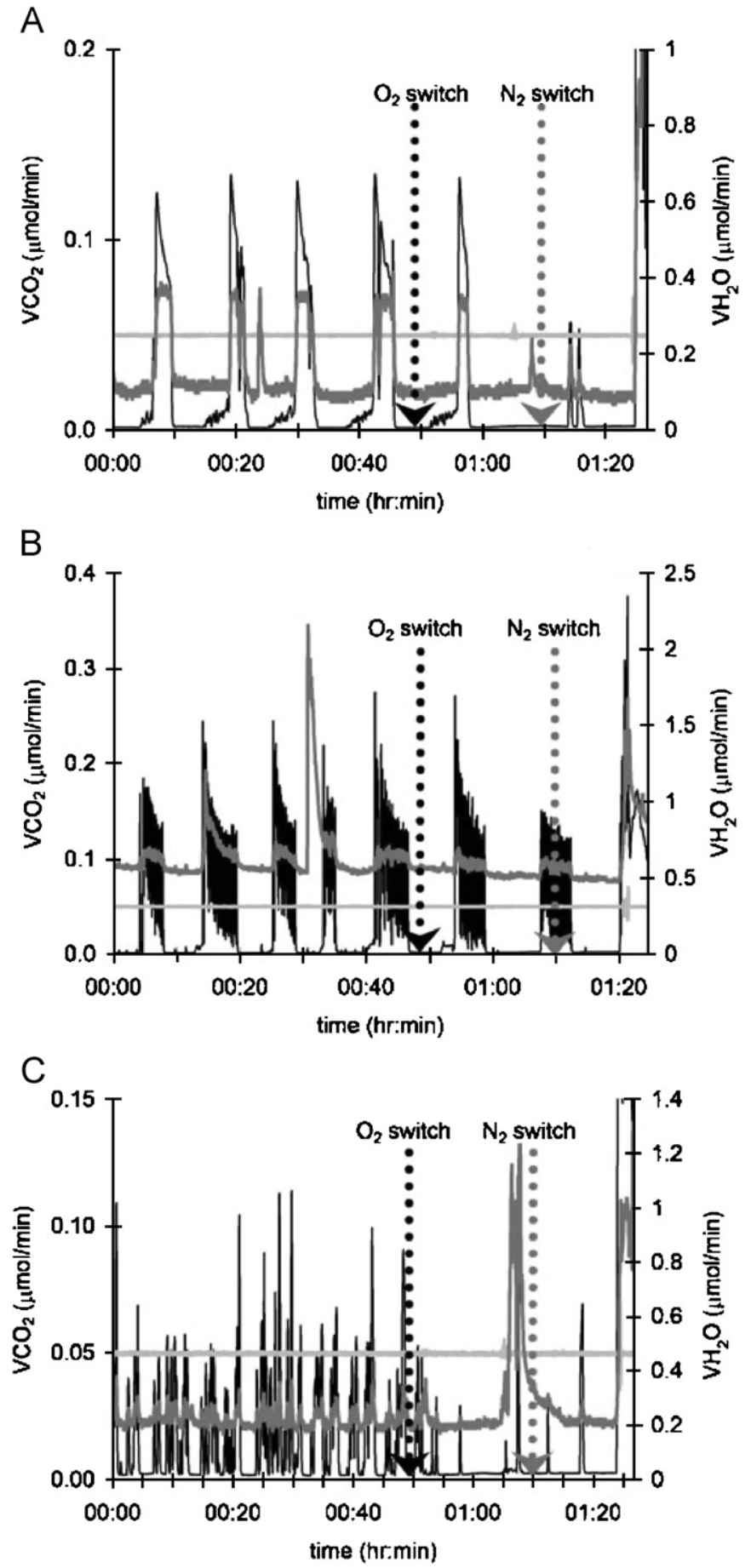

Fig. 1. Representative gas exchange and water loss patterns found in the Perisphaeria species studied here: (A) discontinuous gas exchange cycles, (B) the burst-interburst pattern, (C) continuous gas exchange. Only the last $85 \mathrm{~min}$ of each recording are shown. $\dot{V} \mathrm{CO}_{2}$ : thin black line; $\dot{V} \mathrm{H}_{2} \mathrm{O}$ : thick gray line; activity: thin gray line. Arrows represent the switch from normoxia to hyperoxia (black dotted line and arrow) and the switch from hyperoxia to anoxia (gray dotted line and arrow). Note that in (A) and (C) the large burst following anoxia is cut-off vertically to ensure details of the remainder of the trace are not compromised.

Repeatability, and therefore precision, was typically high for mean $\mathrm{H}_{2} \mathrm{O}$ and for $\mathrm{CWL}$ irrespective of the method used (Table 2). The period used for intercept estimates 
Table 1

The numbers and types of gas exchange patterns found in each of the individuals and their responses to hyperoxia and hypoxia

\begin{tabular}{|c|c|c|c|c|c|c|c|}
\hline Individual & Mass (g) & $\#$ & \# DGC & \# BI & \# Cont & \# hypC-phases & \# Anoxic bursts \\
\hline A & $0.450 \pm 0.012$ & 5 & 4 & 1 & 0 & 4 & 5 \\
\hline B & $0.350 \pm 0.007$ & 5 & 2 & 3 & 0 & 5 & 3 \\
\hline $\mathrm{C}$ & $0.454 \pm 0.009$ & 5 & 3 & 1 & 1 & 5 & 4 \\
\hline $\mathrm{D}$ & $0.365 \pm 0.010$ & 5 & 3 & 1 & 1 & 5 & 4 \\
\hline $\mathrm{E}$ & $0.431 \pm 0.008$ & 5 & 2 & 2 & 1 & 4 & 5 \\
\hline $\mathrm{F}$ & $0.340 \pm 0.010$ & 5 & 3 & 0 & 2 & 4 & 1 \\
\hline $\mathrm{G}$ & $0.422 \pm 0.011$ & 5 & 2 & 1 & 2 & 5 & 5 \\
\hline $\mathrm{H}$ & $0.395 \pm 0.011$ & 5 & 5 & 0 & 0 & 4 & 4 \\
\hline I & $0.339 \pm 0.008$ & 5 & 0 & 4 & 1 & 3 & 3 \\
\hline $\mathrm{J}$ & $0.361 \pm 0.013$ & 5 & 2 & 0 & 3 & 4 & 4 \\
\hline K & $0.503 \pm 0.005$ & 5 & 1 & 0 & 4 & 5 & 5 \\
\hline $\mathrm{L}$ & $0.490 \pm 0.013$ & 5 & 1 & 0 & 4 & 3 & 5 \\
\hline M & $0.519 \pm 0.003$ & 5 & 5 & 0 & 0 & 5 & 4 \\
\hline $\mathrm{N}$ & $0.397 \pm 0.007$ & 5 & 3 & 0 & 2 & 5 & 3 \\
\hline $\mathrm{O}$ & $0.368 \pm 0.009$ & 5 & 3 & 0 & 2 & 5 & 4 \\
\hline Total & & 75 & 39 & 13 & 23 & 66 & 59 \\
\hline
\end{tabular}

Table 2

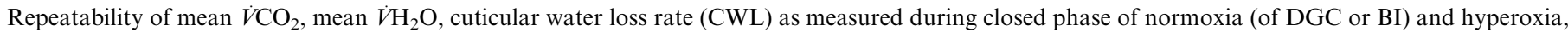
anoxia-induced peak water loss rate (anoxia burst), and slope and $y$-intercept for $\dot{V} \mathrm{CO}_{2}-\dot{V} \mathrm{H}_{2} \mathrm{O}$ regressions obtained over increasing time intervals

\begin{tabular}{|c|c|c|c|c|}
\hline & $F$-ratio (d.f.) & Repeatability \pm S.E. & Confidence interval & $n_{0}$ \\
\hline Mean $\dot{V} \mathrm{CO}_{2}$ & $4.96^{* * *}(14,45)$ & $0.44 \pm 0.13$ & $0.24-0.63$ & 5 \\
\hline Mean $\dot{V} \mathrm{H}_{2} \mathrm{O}$ & $45.87^{* * *}(14,45)$ & $0.90 \pm 0.04$ & $0.82-0.95$ & 5 \\
\hline C-phase $\dot{V} \mathrm{H}_{2} \mathrm{O}$ (traditional method CWL) & $36.21^{* * *}(14,38)$ & $0.88 \pm 0.05$ & $0.83-0.95$ & 3.50 \\
\hline Hyperoxic C-phase $\dot{V} \mathrm{H}_{2} \mathrm{O}$ (hyperoxic estimate CWL) & $55.51^{* * *}(14,36)$ & $0.92 \pm 0.03$ & $0.85-0.96$ & 4.61 \\
\hline RWL (from traditional method) & $0.94(14,38)$ & $0.00 \pm 0.12$ & $0.00-0.19$ & 3.50 \\
\hline RWL (from hyperoxic method) & $4.32^{* * *}(14,34)$ & $0.40 \pm 0.14$ & $0.20-0.62$ & 4.61 \\
\hline RWL (from $y$-intercept method) & $1.53(14,41)$ & $0.09 \pm 0.11$ & $0.00-0.30$ & 4.73 \\
\hline Anoxic $\mathrm{H}_{2} \mathrm{O}$ burst & $1.32(14,44)$ & $0.06 \pm 0.12$ & $0.00-0.28$ & 3.98 \\
\hline$y$-Intercept last $90 \mathrm{~min}$ & $38.30^{* * *}(14,43)$ & $0.88 \pm 0.05$ & $0.83-0.95$ & 3.91 \\
\hline$y$-Intercept last $60 \mathrm{~min}$ & $39.33^{* * *}(14,55)$ & $0.88 \pm 0.05$ & $0.81-0.94$ & 4.73 \\
\hline$y$-Intercept last $30 \mathrm{~min}(y$-intercept estimate CWL) & $39.88^{* * *}(14,56)$ & $0.89 \pm 0.04$ & $0.81-0.94$ & 4.73 \\
\hline$y$-Intercept first $30 \mathrm{~min}$ & $28.00^{* * *}(13,31)$ & $0.84 \pm 0.06$ & $0.80-0.95$ & 3.17 \\
\hline Slope last $90 \mathrm{~min}$ & $10.54^{* * *}(14,30)$ & $0.65 \pm 0.11$ & $0.52-0.84$ & 3.91 \\
\hline Slope last $60 \mathrm{~min}$ & $16.29^{* * *}(14,40)$ & $0.75 \pm 0.08$ & $0.61-0.87$ & 4.73 \\
\hline Slope last $30 \mathrm{~min}$ & $16.51^{* * *}(14,41)$ & $0.76 \pm 0.08$ & $0.61-0.87$ & 4.73 \\
\hline Slope first $30 \mathrm{~min}$ & $2.81^{*}(13,19)$ & $0.27 \pm 0.17$ & $0.07-0.63$ & 3.17 \\
\hline
\end{tabular}

$n_{0}$ represents the average number of observations per individual in the cases where five repeats were not obtained for all individuals.

$* p<0.05 ; * * *<0.001$.

(90, 60, $30 \mathrm{~min}$, or first $30 \mathrm{~min}$ ) had little effect on repeatability of the intercept (or estimate of CWL), but the slope values were more variable if the first $30 \mathrm{~min}$ segment of the last 90 min of a trial was used. Repeatability was significant but lower for estimates of RWL derived from the hyperoxic method, whilst it was barely greater than zero and certainly not significant for RWL derived from the other two methods and for the anoxic $\mathrm{H}_{2} \mathrm{O}$ burst (Table 2). The repeatability of $\dot{V} \mathrm{CO}_{2}$, although significant, was only 0.44 (Table 1 ).

The three methods provided statistically indistinguishable estimates of CWL and, unsurprisingly, CP (Table 3). These estimates of CP are towards the lower end of those found in other insects to date (Fig. 2). However, the hyperoxic method resulted in significantly higher estimates (almost 100\%) of absolute RWL than either of the other methods and, in consequence, substantially higher estimates of \% RWL (Table 3).

$\dot{V} \mathrm{H}_{2} \mathrm{O}$, hypC-phase $\dot{V} \mathrm{H}_{2} \mathrm{O}, \%$ RWL, and the slope and intercept of the $\dot{V} \mathrm{CO}_{2}$ and $\dot{V} \mathrm{H}_{2} \mathrm{O}$ regressions did not differ significantly among the three major gas exchange patterns found in the Perisphaeria sp. in this study (Table 4). However, $\dot{V} \mathrm{CO}_{2}$ differed among the three patterns with continuous $\dot{V} \mathrm{CO}_{2}$ showing the largest value (Table 4). Not unexpectedly, given these results, $\dot{H_{2}} \mathrm{O}, \mathrm{CWL}, \mathrm{RWL}$, $\% \mathrm{CWL}$ and \%RWL found among individuals that had 
Table 3

Cuticular water loss rate (CWL), respiratory water loss rate (RWL), cuticular permeability (CP) and \% RWL as obtained by the three methods examined in this study

\begin{tabular}{|c|c|c|c|c|}
\hline & C-phase $\dot{V} \mathrm{H}_{2} \mathrm{O}$ & hypC-phase $\dot{V} \mathrm{H}_{2} \mathrm{O}$ & $y$-intercept & $p$ \\
\hline RWL $\left(\mu \mathrm{mol} \mathrm{min}^{-1}\right)$ & $0.047 \pm 0.004^{\mathrm{A}}$ & $0.091 \pm 0.008^{\mathrm{B}}$ & $0.053 \pm 0.004^{\mathrm{A}}$ & $<0.0001$ \\
\hline$\%$ RWL & $13.25 \pm 1.61^{\mathrm{A}}$ & $21.84 \pm 1.65^{\mathrm{B}}$ & $14.26 \pm 1.35^{\mathrm{A}}$ & $<0.0001$ \\
\hline
\end{tabular}

Individuals K, L, M were excluded; values \pm S.E.; $p$-values obtained by repeated measures ANOVA followed by Dunnett's post-hoc test. Different letters denote significant differences among methods.

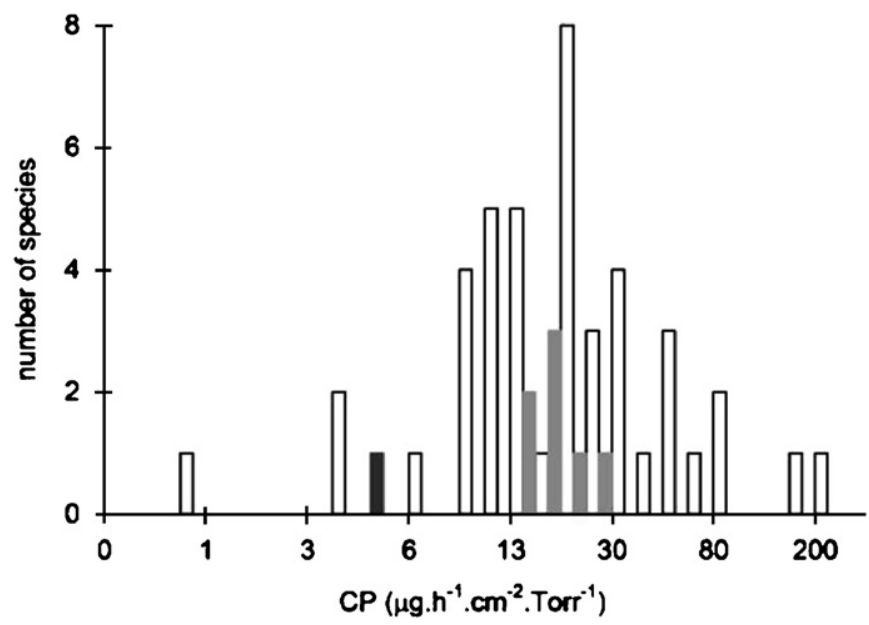

Fig. 2. Frequency distribution of cuticular permeabilities (CP; on a $\log$ scale) obtained from the literature (Hadley, 1994a), from Lighton et al. (2004) and Schilman et al. (2005) (gray bars) and from the Perisphaeria species studied here using the hyperoxic switch method (black bar).

shown both patterns did not differ significantly between continuous and DGE although in this case $\dot{V C O}_{2}$ also differed among the two patterns (Table 5).

\section{Discussion}

\subsection{Gas exchange pattern, metabolic rate and water loss}

The gas exchange patterns and their influence on the absolute values of $\dot{V} \mathrm{CO}_{2}$ found here are similar to those recorded by Marais and Chown (2003). Repeatability estimates of $\mathrm{VCO}_{2}$ also differ little between the studies ( 0.44 here, 0.5 previously) and compare well with those for other insect species (e.g., Chappell and Rogowitz, 2000; Terblanche et al., 2004; Nespolo and Franco, 2007). Given that experimental conditions remained constant across the trials, this work also confirms that the variation in gas exchange pattern among and within individuals is not a consequence of differential flow rates or altered experimental circumstances (see Gray and Bradley, 2006). Therefore, the present work confirms the finding of Marais and Chown (2003) that from a gas exchange perspective this Perisphaeria cockroach is one of the most variable of all insect species that have been investigated to date (Lighton, 1998; Chown, 2001; Marais et al., 2005 provide additional discussion). Nonetheless, this variation can still be classified into a series of recognizable primary patterns. These gas exchange patterns varied to some degree with $\dot{V} \mathrm{CO}_{2}$, a measure of metabolic demand, and to a greater extent than was found in the trials conducted by Marais and Chown (2003).

A relationship between metabolic demand and gas exchange pattern can be interpreted in several ways. It might lead credence to the emergent property hypothesis for the evolution of DGE (Chown and Holter, 2000), it is a prediction of the oxidative damage hypothesis for DGE (Hetz and Bradley, 2005), and the reduction in metabolic rate that accompanies DGE might be thought of as an additional way to effect water savings (Schilman et al., 2005; see also Lehmann, 2001; Addo-Bediako et al., 2001). The results of the present investigation certainly show that respiratory water loss rate increases during anoxia, when the spiracles are opened more widely than under other circumstances (Lighton and Fielden, 1996; Klok et al., 2002; Schilman et al., 2005), therefore suggesting that spiracular control of any form does effect a respiratory water savings (see also Loveridge, 1968; Lighton, 1990; Lighton et al., 1993). However, whether the development of DGE has resulted in additional reduction in respiratory water loss is more controversial. This hygric, or water saving, hypothesis was one of the first explanations proposed to account for evolution of DGE and remains contentious (see Buck et al., 1953; Levy and Schneiderman, 1966; Lighton, 1996; White et al., 2007). Here, the absence of any relationship between RWL or \%RWL and gas exchange pattern, and between $\dot{H}_{2} \mathrm{O}$ and gas exchange pattern provide little support for the hygric hypothesis in this species. Similar results have been found in the other single-species studies that have examined the effect of gas exchange pattern on RWL (Gibbs and Johnson, 2004; Lighton et al., 2004, but see Marais et al., 2005; White et al., 2007 for multi-species studies supporting the hygric hypothesis).

Nonetheless, and for the moment, these outcomes need to be interpreted with caution for several reasons. Foremost among these is the fact that as a consequence of substantial variability in $\dot{V} \mathrm{CO}_{2}$, estimates of respiratory water loss are themselves highly variable. In consequence, only sample sizes much larger than those presently used might reveal an intraspecific relationship between gas 
Table 4

Mean $\dot{V} \mathrm{CO}_{2}, \dot{V} \mathrm{H}_{2} \mathrm{O}$, hypC-phase $\dot{V} \mathrm{H}_{2} \mathrm{O}\left(\mu \mathrm{mol} \mathrm{min}^{-1}\right)$ and \%respiratory water loss, as well as the slopes and intercepts of the regressions of $\dot{V} \mathrm{CO}_{2}$ against $\dot{V} \mathrm{H}_{2} \mathrm{O}$ for different respiratory patterns (individuals $\mathrm{K}, \mathrm{L}, \mathrm{M}$ excluded; values \pm S.E.)

\begin{tabular}{lllllrr}
\hline & $\dot{V} \mathrm{CO}_{2}$ & $\dot{V} \mathrm{H}_{2} \mathrm{O}$ & hypC-phase $\dot{V} \mathrm{H}_{2} \mathrm{O}$ & $\% \mathrm{RWL}$ & Slope & $y$-intercept \\
\hline DGC & $0.025 \pm 0.001^{\mathrm{A}}$ & $0.445 \pm 0.052$ & $0.376 \pm 0.053$ & $20.9 \pm 2.2$ & $1.705 \pm 0.141$ \\
BI & $0.031 \pm 0.003^{\mathrm{B}}$ & $0.655 \pm 0.086$ & $0.521 \pm 0.084$ & $18.9 \pm 3.2$ & $1.534 \pm 0.203$ & $0.390 \pm 0.050$ \\
Cont & $0.036 \pm 0.004^{\mathrm{B}}$ & $0.513 \pm 0.090$ & $0.355 \pm 0.085$ & $26.3 \pm 3.5$ & $1.366 \pm 0.193$ & $0.610 \pm 0.091$ \\
$p$ & 0.025 & 0.572 & 0.755 & 0.374 & 0.339 & 0.479 \\
\hline
\end{tabular}

The significance of differences among gas exchange patterns for each parameter was obtained by REML ANOVA followed by Dunnett's post-hoc test.

Table 5

Comparison of mean $\dot{V} \mathrm{CO}_{2}$, mean $\dot{V} \mathrm{H}_{2} \mathrm{O}$, and absolute and relative cuticular and respiratory water loss rates $\left(\mu\right.$ mol min $\left.{ }^{-1}\right)$ between continuous and discontinuous gas exchange patterns (analysis includes only individuals that performed both types of respiratory patterns; $N=7$; values \pm S.E.)

\begin{tabular}{lllllr}
\hline & $\dot{V} \mathrm{CO}_{2}$ & $\dot{V} \mathrm{H}_{2} \mathrm{O}$ & $\mathrm{RWL}$ & $\mathrm{CWL}$ & $\% \mathrm{RWL}$ \\
\hline Cont & $0.036 \pm 0.004$ & $0.495 \pm 0.095$ & $0.107 \pm 0.016$ & $0.355 \pm 0.085$ & $26.3 \pm 3.5$ \\
DGC & $0.027 \pm 0.001$ & $0.485 \pm 0.071$ & $0.093 \pm 0.014$ & $0.411 \pm 0.073$ & $21.7 \pm 2.7$ \\
$p$ & 0.016 & 0.479 & 0.236 & 0.693 & $73.7 \pm 3.5$ \\
\hline
\end{tabular}

The significance of differences among gas exchange patterns for each parameter was obtained by REML analyses of variance.

exchange pattern and RWL. Indeed, in this particular instance, a post-hoc power analysis for effect size $f=0.25$ at $\alpha=0.05$ revealed a power of 0.32 , meaning that if gas exchange pattern does in fact significantly affect RWL, our analysis only has a $32 \%$ chance of rejecting the null hypothesis of no effect. Increasing this probability to $90 \%$ would require using a total sample size larger than 200 (approximately four times larger than ours). Such sample size effects might account for the differences in outcome between single-species and multi-species investigations of the hygric hypothesis.

\subsection{Precision, bias and accuracy}

Substantial variability in estimates of respiratory water loss suggests initially that not even the most repeatable estimate of RWL (that derived from the hyperoxic switch method) is precise. However, because measured, rather than estimated $\dot{V} \mathrm{CO}_{2}$, showed a similar low repeatability value, it seems more likely that RWL is itself highly variable as a consequence of varying metabolic demand, rather than that the methods are inherently imprecise. Therefore, confirmation of the utility of this method for estimating RWL is also required from a species that shows little variation in gas exchange pattern. Several Namib Desert tenebrionid species seem especially suited for this purpose (see Lighton, 1991).

Nonetheless, comparison of the two major new methods for estimating CWL and RWL and their benchmarking against the third, more traditional DGC (or C-phase) method have provided insight into bias and precision of the methods. For CWL, the methods seem to be equally precise. Moreover, this precision was higher than that found using the regression method in the only other species for which precision has been investigated, the Heelwalker, Karoophasma biedouwensis (Chown et al., 2006b). In this species, a repeatability of 0.55 was found for estimated CWL, whereas measured total water loss rate had a repeatability of 0.75 .

In addition to their varying precision, the methods differed in the bias of their estimates of RWL. The regression method sometimes resulted in negative values of RWL ( $y$-intercept higher than mean $\mathrm{H}_{2} \mathrm{O}$ ), which were generally a consequence of overestimated $\mathrm{H}_{2} \mathrm{O}$ due to small excretory events or the residual water washout from larger ones. Therefore, these $y$-intercept values should be interpreted as error (see also Schilman et al., 2005). This raises the problem of where the cut-off points for erroneous estimates of the $y$-intercept should lie. Clearly $y$-intercept values higher than mean $\dot{V} \mathrm{H}_{2} \mathrm{O}$ should be rejected, but which other values might also be excluded? Further problems confounding the regression method are that the $y$-intercept must be obtained by extrapolating beyond the measured data if the spiracles never close, and that the data points used in the regression are autocorrelated. The former problem can potentially lead to a substantial bias in the estimate (Quinn and Keough, 2002), whilst the latter is always likely to do so because autocorrelation not only affects the significance of least squares regression, but also the parameters estimated using this technique. In other words, adjacent data values are non-independent and a correction must be applied to remove this effect, which may persist across several data points (see Chatfield, 2004). Therefore, and given the typically low estimates of water 
loss provided by the regression method, it must be concluded that this method is the least accurate of the three methods, largely owing to its bias and the likelihood thereof. Nonetheless, it provides a useful first approximation of the contributions of CWL and RWL to total water loss.

The traditional method, because it relies on the closedphase of a discontinuous cycle, cannot be used in the case of continuous gas exchange. Here, the consequent reduction in sample size resulted in a strong decrease in the precision of the method. Nevertheless, the C-phase and hyperoxic switch methods are much less subject to errors resulting from statistical estimation than is the $y$-intercept method. Bootstrap estimation (Quinn and Keough, 2002) supports this conclusion indicating little difference between the bootstrap statistics and those obtained from the original sample (bootstrap data not shown). However, the hyperoxic switch method indicated larger RWL than the C-phase method. This difference between the methods may be the outcome of a prolonged period of spiracular closure during the hyperoxic switch resulting in a much larger estimate of RWL using this method. Quite why this should be the case given non-significant differences in CWL is not clear. However, it seems likely that owing to a slower response of water by comparison with $\mathrm{CO}_{2}$ in the flow-through system we used, the C-phase method consistently overestimated the contribution of CWL to the total water loss rate by comparison with the hyperoxic switch method in which closure of spiracles took place for a prolonged period resulting in a less biased estimate of CWL. One alternative explanation is that the ongoing slight decline in water loss rates across the experiment (see Fig. 1B) lead to an overestimate of RWL. However, an analysis of the decline showed that over the measured period typically used, it amounted to $c$. only c. $20 \%$ of the variation in estimates of RWL. Therefore, it seems unlikely that this source of variation fully explains the differences in RWL. A further alternative is that because the hyperoxic switch method included continuous traces, whereas the C-phase method did not, the former should be higher. Recalculating the RWL values excluding the continuous traces reveals that the hyperoxic switch estimates are $6-8 \%$ lower than when including these traces. Again, this does not fully account for the differences among methods.

The accuracy of the hyperoxic switch method requires measuring $\dot{V} \mathrm{H}_{2} \mathrm{O}$ at a time interval when $\dot{V} \mathrm{CO}_{2}$ is at or near zero, which does not occur after the same length of time in hyperoxia for all individuals. In consequence, CWL is likely to be overestimated when measuring insects in groups rather than individually. Lighton et al. (2004) proposed analytical methods to overcome this source of bias. Therefore, of the three methods we examined for estimating CWL and RWL, the hyperoxic switch method seems most accurate given that it is precise and appears to be relatively unbiased for single insect measurements in its estimate of CWL. Confirmation of this conclusion would require comparison of data derived from this method with those obtained using gravimetric or isotopic techniques, acknowledging that these methods are prone to a different set of biases and have rarely had their precision estimated (reviews in Hadley 1994a; Chown and Nicolson, 2004).

If it is presumed that the DGC C-phase method provides slightly lower estimates of RWL than the hyperoxic switch method, then estimates of \% RWL may well have to be revised slightly upwards. Nonetheless, in this particular species estimates of $\%$ RWL were within the bounds of what has been recorded to date (even if these bounds were to be shifted to the right by several percent). However, the estimates of $\mathrm{CP}$ are low by comparison with other species (Fig. 2), being similar to those recorded for Namib Desert tenebrionids (Hadley and Louw, 1980). Such a low value in this Perisphaeria species is not unrealistic given that in summer the microhabitats occupied by this species are likely to be both dry (mean monthly rainfall for October-March, 1967-2003 in the adjacent Jonkershoek Valley: $31 \mathrm{~mm}$ ), and relatively warm (mean daily microhabitat temperature for November-March, 08:00-20:00 h, 2002-2005, measured hourly using iButton thermochron dataloggers: $23.5^{\circ} \mathrm{C}$ ).

Our final investigation of precision concerned the extent to which an anoxia treatment could provide an estimate either of spiracular opening or tracheal system properties as suggested by Lighton et al. (2004). Observations of the responses to anoxia revealed that in this cockroach species, individuals generally released $\mathrm{CO}_{2}$ and water at low rates for at least several minutes before producing very high and distinct peaks. We consistently measured the value of WLR at the peak but found that repeatability was near zero, indicating that the peak observed is highly variable within individuals. We conclude from this result that anoxic peak WLR cannot be used to estimate spiracular characteristics of insects.

\section{Acknowledgments}

We thank Elrike Marais and John Terblanche for assistance with the animals and with statistical analyses, respectively, and both them and Susan Jackson for reading an earlier draft of this manuscript. Erika Nortje and Elrike Marais provided climate data. Two referees provided useful comments on an earlier version of the manuscript. This work was supported by a Stellenbosch University post-doctoral scholarship to E.M.G., and by National Research Foundation Grant FA2004032000006 to S.L.C.

\section{References}

Addo-Bediako, A., Chown, S.L., Gaston, K.J., 2001. Revisiting water loss in insects: a large scale view. Journal of Insect Physiology 47, 1377-1388.

Alpert, P., 2006. Constraints of tolerance: why are desiccation-tolerant organisms so small or rare? Journal of Experimental Biology 209, $1575-1584$ 
Bech, C., Langseth, I., Gabrielsen, G.W., 1999. Repeatability of basal metabolism in breeding female kittiwakes Rissa tridactyla. Proceedings of the Royal Society of London B 266, 2161-2167.

Becker, W.A., 1984. A Manual of Quantitative Genetics. Academic Enterprises, Pullman, Washington.

Benoit, J.B., Denlinger, D.L., 2007. Suppression of water loss during adult diapause in the northern house mosquito, Culex pipiens. Journal of Experimental Biology 210, 217-226.

Bosch, M., Chown, S.L., Scholtz, C.H., 2000. Discontinuous gas exchange and water loss in the keratin beetle Omorgus radula: further evidence against the water conservation hypothesis? Physiological Entomology 25, 309-314.

Bradley, T.J., Williams, A.E., Rose, M.R., 1999. Physiological responses to selection for desiccation resistance in Drosophila melanogaster. American Zoologist 39, 337-345.

Buck, J., Keister, M., Specht, H., 1953. Discontinuous respiration in diapausing Agapema pupae. Anatomical Record 117, 541.

Chappell, M.A., Rogowitz, G.L., 2000. Mass, temperature and metabolic effects on discontinuous gas exchange cycles in eucalyptus-boring beetles (Coleoptera: Cerambycidae). Journal of Experimental Biology 203, 3809-3820.

Chappell, M.A., Zuk, M., Johnsen, T.S., 1996. Repeatability of aerobic performance in Red Junglefowl: effects of ontogeny and nematode infection. Functional Ecology 10, 578-585.

Chatfield, C., 2004. The Analysis of Time Series. An Introduction. CRC Press, Boca Raton, FL.

Chown, S.L., 2001. Physiological variation in insects: hierarchical levels and implications. Journal of Insect Physiology 47, 649-660.

Chown, S.L., 2002. Respiratory water loss in insects. Comparative Biochemistry and Physiology A 133, 791-804.

Chown, S.L., Davis, A.L.V., 2003. Discontinuous gas exchange and the significance of respiratory water loss in scarabaeine beetles. Journal of Experimental Biology 206, 3547-3556.

Chown, S.L., Holter, P., 2000. Discontinuous gas exchange cycles in Aphodius fossor (Scarabaeidae): a test of hypotheses concerning origins and mechanisms. Journal of Experimental Biology 203, 397-403.

Chown, S.L., Nicolson, S.W., 2004. Insect Physiological Ecology. Mechanisms and Patterns. Oxford University Press, Oxford.

Chown, S.L., Gibbs, A.G., Hetz, S.K., Klok, C.J., Lighton, J.R.B., Marais, E., 2006a. Discontinuous gas exchange in insects: a clarification of hypotheses and approaches. Physiological and Biochemical Zoology 79, 333-343.

Chown, S.L., Marais, E., Picker, M.D., Terblanche, J.S., 2006b. Gas exchange characteristics, metabolic rate and water loss of the Heelwalker, Karoophasma biedouwensis (Mantophasmatodea: Austrophasmatidae). Journal of Insect Physiology 52, 442-449.

Djawdan, M., Chippindale, A.K., Rose, M.R., Bradley, T.J., 1998. Metabolic reserves and evolved stress resistance in Drosophila melanogaster. Physiological Zoology 71, 584-594.

Duncan, F.D., 2003. The role of the subelytral cavity in a tenebrionid beetle, Onymacris multistriata (Tenebrionidae: Adesmiini). Journal of Insect Physiology 49, 339-346.

Duncan, F.D., Krasnov, B., McMaster, M., 2002. Metabolic rate and respiratory gas-exchange patterns in tenebrionid beetles from the Negev Highlands, Israel. Journal of Experimental Biology 205, 791-798.

Edney, E.B., McFarlane, J., 1974. The effect of temperature on transpiration in the desert cockroach, Arenivaga investigata, and in Periplaneta americana. Physiological Zoology 47, 1-12.

Gibbs, A.G., Johnson, R.A., 2004. The role of discontinuous gas exchange in insects: the chthonic hypothesis does not hold water. Journal of Experimental Biology 207, 3477-3482.

Gibbs, A.G., Chippindale, A.K., Rose, M.R., 1997. Physiological mechanisms of evolved desiccation resistance in Drosophila melanogaster. Journal of Experimental Biology 200, 1821-1832.

Gibbs, A.G., Fukuzato, F., Matzkin, L.M., 2003. Evolution of water conservation mechanisms in Drosophila. Journal of Experimental Biology 206, 1183-1192.
Gray, E.M., Bradley, T.J., 2006. Evidence from mosquitoes suggests that cyclic gas exchange and discontinuous gas exchange are two manifestations of a single respiratory pattern. Journal of Experimental Biology 209, 1603-1611.

Hadley, N.F., 1994a. Water Relations of Terrestrial Arthropods. Academic Press, San Diego.

Hadley, N.F., 1994b. Ventilatory patterns and respiratory transpiration in adult terrestrial insects. Physiological Zoology 67, 175-189.

Hadley, N.F., Louw, G.N., 1980. Cuticular hydrocarbons and evaporative water loss in two tenebrionid beetles from the Namib Desert. South African Journal of Science 76, 298-301.

Hetz, S.K., Bradley, T.J., 2005. Insects breathe discontinuously to avoid oxygen toxicity. Nature 433, 516-519.

Hoffmann, A.A., Hallas, R.J., Dean, J.A., Schiffer, M., 2003. Low potential for climatic stress adaptation in a rainforest Drosophila species. Science 301, 100-102.

Kestler, P., 1985. Respiration and respiratory water loss. In: Hoffmann, K.H. (Ed.), Environmental Physiology and Biochemistry of Insects. Springer, Berlin, pp. 137-186.

Klok, C.J., Mercer, R.D., Chown, S.L., 2002. Discontinuous gas-exchange in centipedes and its convergent evolution in tracheated arthropods. Journal of Experimental Biology 205, 1019-1029.

Krebs, C.J., 1999. Ecological Methodology, second ed. Benjamin/ Cummings, Menlo Park.

Lehmann, F.-O., 2001. Matching spiracle opening to metabolic need during flight in Drosophila. Science 294, 1926-1929.

Lessells, C.M., Boag, P.T., 1987. Unrepeatable repeatabilities: a common mistake. Auk 104, 116-121.

Levy, R.I., Schneiderman, H.A., 1966. Discontinuous respiration in insects-II. The direct measurement and significance of changes in tracheal gas composition during the respiratory cycle of silkworm pupae. Journal of Insect Physiology 12, 83-104.

Lighton, J.R.B., 1990. Slow discontinuous ventilation in the Namib dunesea ant Camponotus detritus (Hymenoptera, Formicidae). Journal of Experimental Biology 151, 71-82.

Lighton, J.R.B., 1991. Ventilation in Namib Desert tenebrionid beetles: mass scaling and evidence of a novel quantized flutter-phase. Journal of Experimental Biology 159, 249-268.

Lighton, J.R.B., 1992. Direct measurement of mass loss during discontinuous ventilation in two species of ants. Journal of Experimental Biology 173, 289-293.

Lighton, J.R.B., 1996. Discontinuous gas exchange in insects. Annual Review of Entomology 41, 309-324.

Lighton, J.R.B., 1998. Notes from underground: towards ultimate hypotheses of cyclic, discontinuous gas-exchange in tracheate arthropods. American Zoologist 38, 483-491.

Lighton, J.R.B., Fielden, L.J., 1996. Gas exchange in wind spiders (Arachnida, Solphugidae): independent evolution of convergent control strategies in solphugids and insects. Journal of Insect Physiology 42, 347-357.

Lighton, J.R.B., Garrigan, D.A., Duncan, F.D., Johnson, R.A., 1993 Spiracular control of respiratory water loss in female alates of the harvester ant Pogonomyrmex rugosus. Journal of Experimental Biology 179, 233-244.

Lighton, J.R.B., Schilman, P.E., Holway, D.A., 2004. The hyperoxic switch: assessing respiratory water loss rates in tracheate arthropods with continuous gas exchange. Journal of Experimental Biology 207, 4463-4471.

Loveridge, J.P., 1968. The control of water loss in Locusta migratoria migratorioides R. \& F. II. Water loss through the spiracles. Journal of Experimental Biology 49, 15-29.

Machin, J., Lampert, G.J., 1987. An improved water content model for Periplaneta cuticle: effects of epidermis model and cuticle damage. Journal of Insect Physiology 33, 647-655.

Machin, J., Kestler, P., Lampert, G.J., 1991. Simultaneous measurements of spiracular and cuticular water losses in Periplaneta americana: implications for whole-animal mass loss studies. Journal of Experimental Biology 161, 439-453. 
Marais, E., Chown, S.L., 2003. Repeatability of standard metabolic rate and gas exchange characteristics in a highly variable cockroach, Perisphaeria sp. Journal of Experimental Biology 206, 4565-4574.

Marais, E., Klok, C.J., Terblanche, J.S., Chown, S.L., 2005. Insect gas exchange patterns: a phylogenetic perspective. Journal of Experimental Biology 208, 4495-4507.

Nespolo, R.F., Franco, M., 2007. Whole-animal metabolic rate is a repeatable trait: meta-analysis. Journal of Experimental Biology 210, 2000-2005.

Quinlan, M.C., Hadley, N.F., 1993. Gas exchange, ventilatory patterns, and water loss in two lubber grasshoppers: quantifying cuticular and respiratory transpiration. Physiological Zoology 66, 628-642.

Quinlan, M.C., Lighton, J.R.B., 1999. Respiratory physiology and water relations of three species of Pogonomyrmex harvester ants (Hymenoptera: Formicidae). Physiological Entomology 24, 293-302.

Quinn, G.P., Keough, M.J., 2002. Experimental Design and Data Analysis for Biologists. Cambridge University Press, Cambridge.

Rourke, B.C., 2000. Geographic and altitudinal variation in water balance and metabolic rate in a California grasshopper, Melanoplus sanguinipes. Journal of Experimental Biology 203, 2699-2712.

Schilman, P.E., Lighton, J.R.B., Holway, D.A., 2005. Respiratory and cuticular water loss in insects with continuous gas exchange: comparison across five ant species. Journal of Insect Physiology 51, $1295-1305$.

Shelton, T.G., Appel, A.G., 2001. Cyclic $\mathrm{CO}_{2}$ release and water loss in alates of the eastern subterranean termite (Isoptera: Rhinotermitidae). Annals of the Entomological Society of America 94, 420-426.

Telonis-Scott, M., Guthridge, K.M., Hoffmann, A.A., 2006. A new set of laboratory-selected Drosophila melanogaster lines for the analysis of desiccation resistance: response to selection, physiology and correlated responses. Journal of Experimental Biology 209, 1837-1847.

Terblanche, J.S., Klok, C.J., Chown, S.L., 2004. Metabolic rate variation in Glossina pallidipes (Diptera: Glossinidae): gender, ageing and repeatability. Journal of Insect Physiology 50, 419-428.

Walther, B.A., Moore, J.L., 2005. The concepts of bias, precision and accuracy, and their use in testing the performance of species richness estimators, with a literature review of estimator performance. Ecography 28, 815-829.

White, C.R., Blackburn, T.M., Terblanche, J.S., Marais, E., Gibernau, M., Chown, S.L., 2007. Evolutionary responses of discontinuous gas exchange in insects. Proceedings of the National Academy of Sciences of the USA 104, 8357-8361.

Zachariassen, K.E., Anderson, J., Maloiy, G.M.O., Kamau, J.M.Z., 1987. Transpiratory water loss and metabolism of beetles from arid areas in East Africa. Comparative Biochemistry and Physiology A 86, 403-408. 